\title{
NON-LINEAR STOCHASTIC DYNAMICS OF A CABLE-MASS SYSTEM WITH FINITE BENDING STIFFNESS VIA THE EQUIVALENT LINEARIZATION TECHNIQUE
}

\author{
HANNA WEBER \\ West Pomeranian University of Technology, Faculty of Civil Engineering and Architecture, Szczecin, Poland \\ e-mail: hanna.weber@zut.edu.pl \\ STEFAN KACZMARCZYK \\ University of Northampton, Faculty of Arts, Science and Technology, UK \\ e-mail: stefan.kaczmarczyk@northampton.ac.uk \\ RADOSŁAW IWANKIEWICZ \\ West Pomeranian University of Technology, Faculty of Civil Engineering and Architecture, Szczecin, Poland \\ e-mail: radoslaw.iwankiewicz@zut.edu.pl
}

\begin{abstract}
The non-linear stochastic dynamic behaviour of a high-rise vertical transportation system modelled as a concentrated mass and a cable with finite bending stiffness is considered. The slow time scale is defined and lateral cable displacements coupled with transverse motions are expanded in terms of approximating functions. The excitation of the high-rise building is assumed in the form of a narrow-band mean-square process equivalent to the harmonic process. The equivalent linearization technique is used to replace the original non-linear system with a linear approximation whose coefficients are determined from minimization of the mean-square equation difference between both systems.
\end{abstract}

Keywords: stochastic dynamics, nonlinear systems, cable, finite bending stiffness, equivalent linearization technique

\section{Introduction}

The equations and numerical results included in this paper concern the problem presented at the 4th Polish Congress of Mechanics and 23rd Conference on Computer Methods in Mechanics (PCM-CMM) which took place in September 2019.

From the point of view of safety and structural integrity of a system, it is very important to consider vibrations (sway) of high-rise buildings due to dynamic excitations. The sway with low frequencies and large amplitudes is observed (Kijewski-Correa and Pirnia, 2007) which affects dynamic behaviour of structural parts of vertical transportation systems such as cables or ropes deployed in lift installations. The longitudinal time-dependent displacements of a cable-mass system are coupled with its transverse motions. Therefore, dynamic behaviour of the system is described by non-linear non-stationary equations of motion. In literature, different approaches can be found to represent the excitation mechanism in analysis of high-rise buildings under wind loads - from deterministic functions to stochastic methods (Larsen et al., 2007; Kaczmarczyk et al., 2009; Giaccu et al., 2015; Kaczmarczyk and Iwankiewicz, 2017a). Undoubtedly, the real nature of forces caused by wind is randomness. The corresponding excitation mechanism can be considered as random. The governing equations are then expressed by linear differential equations that define the excitation as a narrow-band random process.

It is difficult to use analytical methods to solve nonstationary and non-linear equations (Terumichi et al., 1995). Therefore, in most cases, numerical techniques are applied. In this 
paper, the earlier research concerning vibration of a cable-mass system with time-varying length in high-rise buildings carried out by Kaczmarczyk and Iwankiewicz $(2017 \mathrm{a}, \mathrm{b})$ and Weber et al. (2019), where the string model of the cable (rope) with concentrated mass was considered is developed further. The rope considered as a long beam of small bending stiffness is examined, which requires modification of deterministic and stochastic equations. To replace the original non-linear set of equations by an equivalent linear one, an equivalent (statistical) linearization technique is applied. Using the condition of mean-square minimization of the error between the non-linear and linear system, the coefficients of the equivalent linear system in terms of expectations of particular random state variables are obtained.

The equivalent statistical linearization technique has been implemented to consider a broad range of problems in the area of non-linear stochastic dynamics (Caughey, 1963; Spanos, 1981; Roberts and Spanos, 1990; Socha, 2008). In many papers, the effectiveness of this technique is compared with other statistical methods (Roberts, 1981; Proppe et al., 2003) or combined with other computational methods in random vibrations (Spanos and Evangelatos, 2010; Kougioumtzoglou et al., 2017). In this work, the equivalent linearization technique is used to obtain expectations and variances of generalized coordinates describing dynamic behaviour of the cable and of the main mass. The results are then compared with the values determined from the original non-linear system subjected to the deterministic harmonic kinematic excitation and with the results generated by the application of Monte Carlo (MC) simulation.

\section{Non-linear deterministic system}

Consider a high-rise building with an elevator car moving vertically in a lift shaft with the transport speed and acceleration denoted as $V(t)$ and $a(t)=\dot{V}(t)$, respectively (Fig. 1a,b).

(a)

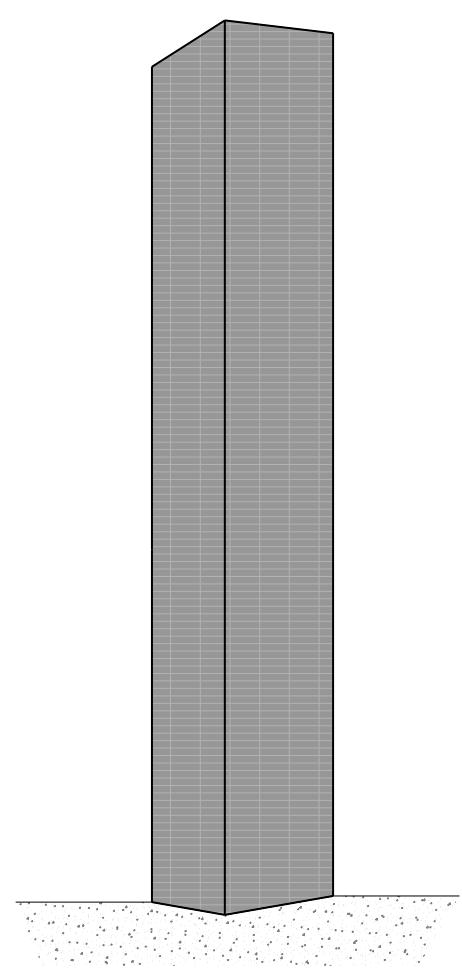

(b)

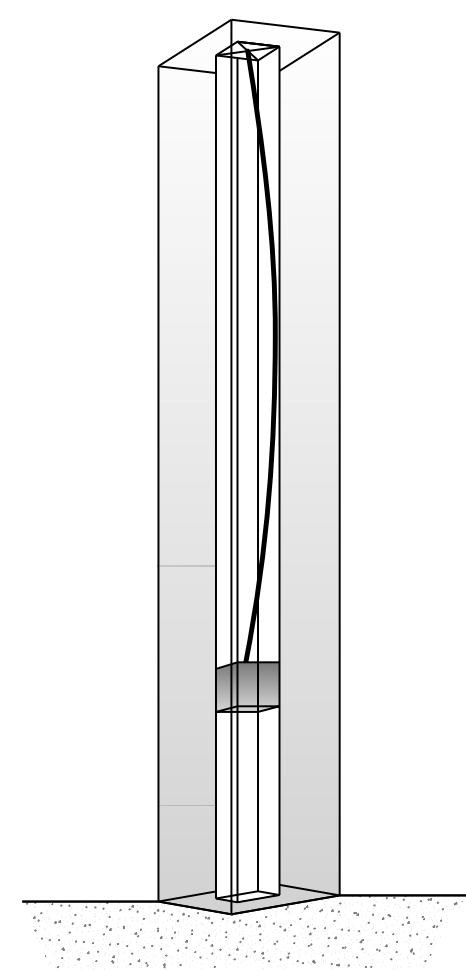

(c)

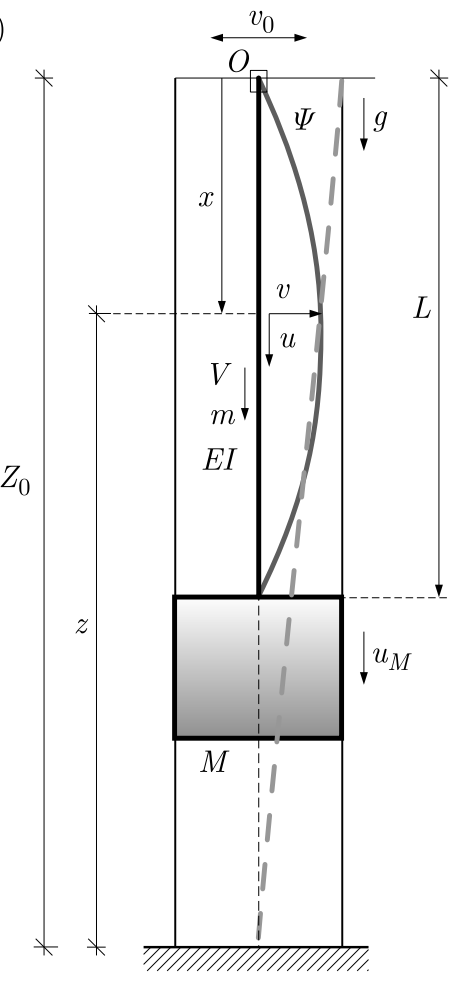

Fig. 1. (a) High-rise building; (b) lift elevator; (c) lift car-cable system schematic model 
The external dynamic loads cause the sway of the building structure. As a result, lateral displacements at the top of the host structure denoted as $v_{0}$ are observed, which leads to excitation of the car suspension ropes (cables) deployed within the system. The longitudinal displacements of the rope $u(x, t)$ are coupled with its lateral vibrations $v(x, t)$. Often the cables are considered as strings without bending stiffness. However, in lift applications, the cables are steel wire ropes (SWR) of a stranded construction of a finite low (non-zero) bending stiffness (Kaczmarczyk and Mirhadizadeh, 2016). The model considered here comprises the concentrated mass $M$ moving slowly downwards. The mass is attached at the lower end of the cable of bending stiffness $E I$ and the longitudinal stiffness $E A$, respectively (see Fig. 1c). The longitudinal (axial) vibrations of the main mass are represented by $u_{M}(t)$. In this model $E, A, I$ and $m$ denote the modulus of elasticity, cross-sectional metallic area, second moment of inertia of the cross-sectional area and mass per unit length. During the overall transport motion, length of the cable varies with time and is represented as $L=L(t)$.

The cable strain measure is defined as $\varepsilon(x, t)=u_{x}(x, t)+0.5 v_{x}^{2}(x, t)$, while its quasi-static tension is expressed by the formula $T(t)=(M+m L(t))(g-a(t))$. The partial derivative with respect to $x$ is denoted as $(\cdot)_{x}$. If the Hamilton principle is applied, the equation governing the dynamic response of the system can be derived from in the following form

$$
\int_{t_{1}}^{t_{2}}\left(\delta E(x, t)-\delta \Pi(x, t)+\delta W_{n c}(x, t)\right)=0 \quad \delta W_{n c}(x, t)=0 \quad \text { at } \quad t=t_{1}, t_{2}
$$

with $E(x, t), \Pi(x, t), W_{n c}(x, t)$ being the kinetic and potential energies of the system and the work of external non-conservative forces, respectively. The kinetic energy of the presented system is expressed by

$$
E(x, t)=\int_{0}^{L} \frac{1}{2} m\left[(\dot{u}(x, t)+V(t))^{2}+\dot{v}^{2}(x, t)\right] d x+\frac{1}{2} M\left(\dot{u}_{M}(t)+V(t)\right)^{2}
$$

where the first term represents kinetic energy density of the cable while the second one kinetic energy of the end mass. The total derivatives with respect to time are denoted as $\dot{u}(x, t) \equiv$ $d u(x, t) / d t=u_{t}(x, t)+V(t) u_{x}(x, t)$ and $\dot{v}(x, t) \equiv d v(x, t) / d t=v_{t}(x, t)+V(t) v_{x}(x, t)$. If the potential energy is adopted in the form

$$
\Pi(x, t)=\int_{0}^{L}\left[\frac{1}{2} E I v_{x x}^{2}(x, t)+T^{i}(x, t) \varepsilon(x, t)+\frac{1}{2} E A \varepsilon^{2}(x, t)-m g u(x, t)\right] d x-M g u_{M}(t)
$$

and the total derivative is expressed by

$$
\frac{d^{2}(\cdot)}{d t^{2}}=(\cdot)_{t t}+2 V(\cdot)_{x t}+V^{2}(\cdot)_{x x}+a(\cdot)_{x}
$$

the following set of partial differential equations of motion is then obtained as

$$
\begin{aligned}
& m \frac{d^{2} u(x, t)}{d t^{2}}-E A \varepsilon_{x}(x, t)=0 \\
& m \frac{d^{2} v(x, t)}{d t^{2}}+E I v_{x x x x}(x, t)-T(t) v_{x x}(x, t) \\
& \quad+m(g-a(t))\left(x v_{x x}(x, t)+v_{x}(x, t)\right)-E A\left(\varepsilon(x, t) v_{x}(x, t)\right)_{x}=0 \\
& M \ddot{u}_{M}(t)+\left.E A \varepsilon(x, t)\right|_{x=L}=0
\end{aligned}
$$


where the mean quasi-static tension inside the cable is defined by the formula

$$
T^{i}(x, t)=[M+m(L(t)-x)](g-a(t))
$$

The boundary conditions are assumed as $u(0, t)=0$ and $u_{M}=u(L, t)$ at $x=L$. In further considerations, the longitudinal inertia of the cable in the first part of Eqs. (2.5) is neglected because of significantly higher values of longitudinal frequencies obtained for tensioned cables in comparison to the lateral frequencies and excitation frequencies. Integrating this equation leads to obtaining the following expressions

$$
u_{x}(x, t)=e(t)-\frac{1}{2} v_{x}^{2}(x, t) \quad e(t)=\frac{u_{M}(t)}{L(t)}+\frac{1}{2 L(t)} \int_{0}^{L} v_{x}^{2}(x, t) d x
$$

where $e(t)$ denotes the mean quasi-static axial strain in the rope. The second expression in Eq. (2.7) is derived by integrating the first one over $x$ from 0 to L. Using Eq. (2.4) and Eq. (2.7) leads to reducing Eq. (2.5) to a set of two equations

$$
\begin{aligned}
& m v_{t t}(x, t)+E I v_{x x x x}(x, t)+\left\{m\left(V^{2}(t)+(g-a(t)) x\right)-T(t)\right. \\
& \left.-\frac{E A}{L(t)}\left(u_{M}(t)+\frac{1}{2} \int_{0}^{L} v_{x}^{2}(x, t) d x\right)\right\} v_{x x}(x, t)+m g v_{x}(x, t)+2 m V(t) v_{x t}(x, t)=0 \\
& M \ddot{u}_{M}(t)+\frac{E A}{L(t)}\left(u_{M}(t)+\frac{1}{2} \int_{0}^{L} v_{x}^{2}(x, t) d x\right)=0
\end{aligned}
$$

Due to the slenderness of high-rise buildings, dynamic wind loads cause the buildings to sway. The building can be treated as a cantilever structure subjected bending deformations. Therefore, during the analysis the fundamental mode is assumed in the form of a polynomial shape function, defined as $\Psi(\eta(x))=3 \eta^{2}(x)-2 \eta^{3}(x)$ (Thompson, 1993). If $z(x)=Z_{0}-x$ denotes the coordinate measured from the ground level and $Z_{0}$ is the total height of the entire system, the variable $\eta(x)$ is adopted in the form $\eta(x)=z(x) / Z_{0}$. In nonlinear deterministic solution, the vibrations that are induced at the top end of the cable are assumed in form of harmonic motion $v_{0}(t)$ with the amplitude and frequency denoted as $A_{0}$ and $\Omega_{0}$, respectively. The dynamic lateral displacements at the lower end of the cable can be then given by the following expression

$$
v_{L}(L(t), t)=\Psi_{L}(L(t)) v_{0}(t) \quad \Psi_{L}(L(t))=\Psi\left(\frac{Z_{0}-L(t)}{Z_{0}}\right)
$$

where $\Psi_{L}(L(t))$ represents the polynomial shape function evaluated at $x=L(t)$. Because of the small change of the cable length $L(t)$ during the period $T_{0}$ corresponding to the fundamental frequency of the system $f_{0}$ (Evan-Iwanowski, 1976; Mitropolskii, 1965), a slow time scale can be introduced as $\tau=\epsilon t$. If $t_{0}$ is a given time instant corresponding to the fundamental frequency and $L_{0}=L\left(t_{0}\right)$, a small parameter $\epsilon \ll 1$ can be defined by the equation $\epsilon=\dot{L}\left(t_{0}\right) / f_{0} L_{0}$ (Kaczmarczyk and Iwankiewicz, 2017b). This slow time scale allows one to differentiate the slow varying functions from fast varying ones. Using $L=L(\tau)$ and a single-mode approximation, the overall lateral displacements of the cable can be assumed in the form of the finite series as (Kaczmarczyk, 1997)

$$
v(x, t ; \tau)=\bar{v}(x, t ; \tau)+\left(1+\frac{\Psi_{L}(L(\tau))-1}{L(\tau)} x\right) v_{0}(t)
$$


with

$$
\bar{v}(x, t ; \tau)=\sum_{n=1}^{N} \Phi_{n}(x ; L(\tau)) q_{n}(t)
$$

where $\bar{v}(x, t ; \tau)$ are relative lateral displacements that satisfy the homogenous boundary conditions. When $N$ and $q_{n}(t)$ denote the number of modes taken into consideration and the fast varying coefficients of expansion (generalized coordinates), respectively, the slow varying orthogonal trial functions are given by the expression $\Phi_{n}(x, L(\tau))=\sin (n \pi / L(\tau)) x, n=1,2, \ldots, N$, and satisfy the homogenous boundary conditions. Using Eq. (2.10) and partial derivatives of lateral displacements with respect to time and $x$ defined by

$$
\begin{aligned}
& v_{t t}(x, t ; \tau)=\bar{v}_{t t}(x, t ; \tau)+\left(1+\frac{\Psi_{L}(L(\tau))-1}{L(\tau)} x\right) \ddot{v}_{0}(t) \\
& v_{x}(x, t ; \tau)=\bar{v}_{x}(x, t ; \tau)+\frac{\Psi_{L}(L(\tau))-1}{L(\tau)} v_{0}(t) \\
& v_{x t}(x, t ; \tau)=\bar{v}_{x t}(x, t ; \tau)+\frac{\Psi_{L}(L(\tau))-1}{L(\tau)} \dot{v}_{0}(t)
\end{aligned}
$$

Eqs.(2.8) are rewritten in the following form

$$
\begin{aligned}
& m \bar{v}_{t t}(x, t ; \tau)+E I \bar{v}_{x x x x}(x, t ; \tau)+m g \bar{v}_{x}(x, t ; \tau)+2 m V(\tau) \bar{v}_{x t}(x, t ; \tau) \\
& \quad+m\left(1+\frac{\Psi_{L}(L(\tau))-1}{L(\tau)} x\right) \ddot{v}_{0}(t)+2 m V(\tau) \frac{\Psi_{L}(L(\tau))-1}{L(\tau)} \dot{v}_{0}(t)+m g \frac{\Psi_{L}(L(\tau))-1}{L(\tau)} v_{0}(t) \\
& \quad+\left\{-T(\tau)+m\left[V^{2}(\tau)+(g-a(\tau)) x\right]-\frac{E A}{L(\tau)}\left[u_{M}(t)+\frac{1}{2} \sum_{n=1}^{N} \frac{n^{2} \pi^{2}}{2 L(\tau)} q_{n}^{2}(t)\right.\right. \\
& \left.\left.\quad+\frac{1}{2} \frac{\left(\Psi_{L}(L(\tau))-1\right)^{2}}{L(\tau)} v_{0}^{2}(t)\right]\right\} \bar{v}_{x x}(x, t ; \tau)=0 \\
& M \ddot{u}_{M}(t)+\frac{E A}{L(\tau)}\left(u_{M}(t)+\frac{1}{2} \int_{0}^{L} v_{x}^{2}(x, t ; \tau) d x\right)=0
\end{aligned}
$$

The expression for the eigenvalue is given by

$$
\bar{\omega}_{r}^{2}(\tau)=\left(\frac{r \pi}{L(\tau)}\right)^{4} \frac{E I}{m}\left[1+\frac{T(\tau)}{E I}\left(\frac{L(\tau)}{r \pi}\right)^{2}\right]=\lambda_{r}^{2}(\tau)\left[\hat{c}_{r}^{2}(\tau)+\bar{c}^{2}(\tau)\right]
$$

where

$$
\lambda_{r}(\tau)=\frac{r \pi}{L(\tau)} \quad \bar{c}^{2}(\tau)=\frac{T(\tau)}{m} \quad \widehat{c}_{r}^{2}(\tau)=\lambda_{r}^{2}(\tau) \frac{E I}{m}
$$

Using Eq. (2.13) and the orthogonality conditions defined as

$$
\int_{0}^{L} \Phi_{n} \Phi_{r} d x=\left\{\begin{array}{ll}
\frac{L}{2} & n=r \\
0 & n \neq r
\end{array} \quad \int_{0}^{L} \Phi_{n}^{\prime \prime} \Phi_{r} d x= \begin{cases}-\frac{r^{2} \pi^{2}}{2 L} & n=r \\
0 & n \neq r\end{cases}\right.
$$

where the prime denotes differentiation with respect to $x$, the set of differential equations of motion given by Eq. (2.12) is then transformed to the form $(r, n=1,2, \ldots, N)$ 


$$
\begin{aligned}
& m_{r}(\tau) \ddot{q}_{r}(t)+m_{r}(\tau) \bar{\omega}_{r}^{2}(\tau) q_{r}(t)+m V^{2}(\tau)\left(-\frac{r^{2} \pi^{2}}{2 L(\tau)}\right) q_{r}(t)+m(g-a(\tau)) \sum_{n} \Theta_{r n} q_{n}(t) \\
& +m g \sum_{n} \Psi_{r n} q_{n}(t)+2 m V(\tau) \sum_{n} \Psi_{r n} \dot{q}_{n}(t)+m \ddot{v}_{0}(t)\left(\chi_{r}(\tau)+\frac{\Psi_{L}(L(\tau))-1}{L(\tau)} \Pi_{r}(\tau)\right) \\
& +2 m V(\tau) \frac{\Psi_{L}(L(\tau))-1}{L(\tau)} \dot{v}_{0}(t) \chi_{r}(\tau)+m g \frac{\Psi_{L}(L(\tau))-1}{L(\tau)} v_{0}(t) \chi_{r}(\tau) \\
& \quad-\frac{E A}{L(\tau)}\left(u_{M}(t)+\frac{1}{2} \sum_{n} \frac{n^{2} \pi^{2}}{2 L(\tau)} q_{n}^{2}(t)+\frac{1}{2} \frac{\left[\Psi_{L}(L(\tau))-1\right]^{2}}{L(\tau)} v_{0}^{2}(t)\right)\left(-\frac{r^{2} \pi^{2}}{2 L(\tau)}\right) q_{r}(t)=0 \\
& \ddot{u}_{M}(t)+\omega_{M}^{2}(\tau) u_{M}(t)+\frac{E A}{M} \sum_{n=1}^{N} \beta_{n}^{2}(\tau) q_{n}^{2}(t)=-\frac{E A}{2 M}\left(\frac{\Psi_{L}(L(\tau))-1}{L(\tau)}\right)^{2} v_{0}^{2}(t)
\end{aligned}
$$

where the following expressions are used

$$
\begin{aligned}
& \Psi_{r n}=\int_{0}^{L} \Phi_{n}^{\prime} \Phi_{r} d x= \begin{cases}0 & n=r \\
\frac{n r}{n^{2}-r^{2}}\left[(-1)^{r+n}-1\right] & n \neq r\end{cases} \\
& \Theta_{r n}=\int_{0}^{L} x \Phi_{n}^{\prime \prime} \Phi_{r} d x= \begin{cases}-\frac{r^{2} \pi^{2}}{4} & n=r \\
-\frac{2 r n^{3}}{\left(n^{2}-r^{2}\right)^{2}}\left[(-1)^{r+n}-1\right] & n \neq r\end{cases} \\
& \chi_{r}(\tau)=\int_{0}^{L} \Phi_{r} d x=-\frac{\left[(-1)^{r}-1\right] L(\tau)}{r \pi} \quad \Pi_{r}(\tau)=\int_{0}^{L} x \Phi_{r} d x=-\frac{(-1)^{r} L^{2}(\tau)}{r \pi} \\
& m_{r}(\tau)=\frac{m L(\tau)}{2} \quad \beta_{n}^{2}(\tau)=\frac{r^{2} \pi^{2}}{4 L^{2}(\tau)} \quad \omega_{M}(\tau)=\sqrt{\frac{E A}{M L(\tau)}}
\end{aligned}
$$

The undamped natural frequency of the main mass is denoted as $\omega_{M}(\tau)$ (in the vertical direction). After taking into account the modal damping ratios $\zeta_{M}, \zeta_{r}$ and noting that $c^{2}(\tau)=$ $E A / m_{r}(\tau)$, the set of Eqs. (2.15) is simplified as

$$
\begin{aligned}
& \ddot{q}_{r}(t)+2 \zeta_{r} \omega_{r}(\tau) \dot{q}_{r}(t)+\lambda_{r}^{2}(\tau)\left[\hat{c}_{r}^{2}(\tau)+\bar{c}^{2}(\tau)-V^{2}(\tau)\right] q_{r}(t)+\sum_{n} K_{r n}(\tau) q_{n}(t) \\
& +\sum_{n} C_{r n}(\tau) \dot{q}_{n}(t)+\lambda_{r}^{2}(\tau) c^{2}(\tau)\left[\frac{u_{M}(t)}{L(\tau)}+\sum_{n} \beta_{n}^{2}(\tau) q_{r}^{2}(t)\right. \\
& \left.+\frac{1}{2}\left(\frac{\Psi_{L}(L(\tau))-1}{L(\tau)}\right)^{2} v_{0}^{2}(t)\right] q_{r}(t)=Q_{r}(t ; \tau) \quad r, n=1,2, \ldots, N \\
& \ddot{u}_{M}(t)+2 \zeta_{M} \omega_{M}(\tau) \dot{u}_{M}(t)+\omega_{M}^{2}(\tau) u_{M}(t)+\frac{E A}{M} \sum_{n=1}^{N} \beta_{n}^{2}(\tau) q_{n}^{2}(t) \\
& =-\frac{E A}{2 M}\left(\frac{\Psi_{L}(L(\tau))-1}{L(\tau)}\right)^{2} v_{0}^{2}(t)
\end{aligned}
$$

where $\omega_{r}(\tau)$ is the undamped natural lateral frequency of the whole system while the coefficients of stiffness and damping denoted as $K_{r n}(\tau)$ and $C_{r n}(\tau)$ together with the modal excitation functions $Q_{r}(x t ; \tau)$ are assumed by the following equations (Kaczmarczyk and Iwankiewicz, 2017b) 


$$
\begin{gathered}
K_{r n}(\tau)= \begin{cases}\frac{L(\tau)}{2}(a(\tau)-g) \lambda_{r}^{2}(\tau) & n=r \\
\frac{2}{L(\tau)}\left(g \frac{n r}{n^{2}-r^{2}}+(a(\tau)-g) \frac{2 r n^{3}}{\left(n^{2}-r^{2}\right)^{2}}\right)\left[(-1)^{r+n}-1\right] & n \neq r\end{cases} \\
C_{r n}(\tau)=\frac{4 V(\tau)}{L(\tau)} \begin{cases}0 & n=r \\
\frac{n r}{n^{2}-r^{2}}\left[(-1)^{r+n}-1\right] & n \neq r\end{cases} \\
Q_{r}(t ; \tau)=-\frac{2}{L(\tau)}\left[\ddot{v}_{0}(t)\left(\chi_{r}(\tau)+\frac{\Psi_{L}(t(\tau))-1}{L(\tau)} \Pi_{r}(\tau)\right)\right. \\
\left.+2 V(\tau) \frac{\Psi_{L}(L(\tau))-1}{L(\tau)} \dot{v}_{0}(t) \chi_{r}(\tau)+g \frac{\Psi_{L}(L(\tau))-1}{L(\tau)} v_{0}(t) \chi_{r}(\tau)\right]
\end{gathered}
$$

The whole vector $\mathbf{q}(t)=\left[q_{1}(t), q_{2}(t), \ldots, q_{N}(t)\right]$ consists of the fast varying (and observed on the $t$ time scale) generalized coordinates corresponding to the lateral modes.

The behaviour of the system in the resonance region, when the fundamental frequency of the system reaches the value that is near the excitation frequency, is now described by an approximated single-mode model given by the expression $(r=1,2, \ldots, N)$

$$
\begin{aligned}
& \ddot{q}_{r}(t)+C_{r}(\tau) \dot{q}_{r}(t)+\frac{\lambda_{r}^{4}(\tau)}{4} c^{2}(\tau) q_{r}^{3}(t)+\frac{2}{L(\tau)} \gamma^{(2)}(\tau) \ddot{v}_{0}(t) \\
& \quad+\frac{4 V(\tau)}{L(\tau)} \gamma^{(1)}(\tau) \chi_{r}(\tau) \dot{v}_{0}(t)+\frac{2 g}{L(\tau)} \gamma^{(1)}(\tau) \chi_{r}(\tau) v_{0}(t)+\lambda_{r}^{2}(\tau)\left[\widehat{c}_{r}^{2}(\tau)+\bar{c}^{2}(\tau)-V^{2}(\tau)\right. \\
& \left.\quad+\frac{L(\tau)}{2}(a(\tau)-g)+c^{2}(\tau)\left(\frac{u_{M}(t)}{L(\tau)}+\frac{1}{2}\left(\gamma^{(1)}(\tau)\right)^{2} v_{0}^{2}(t)\right)\right] q_{r}(t)=0 \\
& \ddot{u}_{M}(t)+2 \zeta_{M} \omega_{M}(\tau) \dot{u}_{M}(t)+\omega_{M}^{2}(\tau) u_{M}(t)+\frac{E A}{M} \frac{\lambda_{r}^{2}(\tau)}{4} q_{r}^{2}(t)+\frac{E A}{2 M}\left(\gamma^{(1)}(\tau)\right)^{2} v_{0}^{2}(t)=0
\end{aligned}
$$

where

$$
\gamma^{(1)}(\tau)=\frac{\Psi_{L}(L(\tau))-1}{L(\tau)} \quad \gamma^{(2)}(\tau)=\chi_{r}(\tau)+\gamma^{(1)}(\tau) \Pi_{r}(\tau) \quad C_{r}(\tau)=2 \zeta_{r} \omega_{r}(\tau)
$$

\section{Stochastic model}

In the deterministic system, the external excitation is assumed in the form of harmonic motion. However, the real nature of the wind load is nondeterministic and, therefore, it should be idealized as a random process. To compare results obtained from both approaches, the excitation in the second one is assumed as a narrow-band mean-square process equivalent to the harmonic process. To satisfy the condition about the continuity and double differentiability, the motion $v_{0}$ is assumed to be the response to the second-order auxiliary filter of the process $(t)$ which, in turn, is the response to the first-order filter of the Gaussian white noise excitation $\xi$ (Larsen et al., 2007). Therefore, the governing equations are adopted in the following form

$$
\begin{aligned}
& \ddot{v}_{0}(t)+2 \zeta_{f} \Omega_{0} \dot{v}_{0}(t)+\Omega_{0}^{2} v_{0}(t)=X(t) \\
& \dot{X}(t)+\alpha X(t)=\alpha \sqrt{2 \pi S_{0}} \xi(t)
\end{aligned}
$$

with the auxiliary filter damping ratio, the white noise power spectrum level and the filter variable denoted as $\zeta_{f}, S_{0}$ and $\alpha$, respectively. For the stochastic process $v_{0}(t)$ to be mean-square 
equivalent to the deterministic harmonic one with the amplitude $A_{0}$, the following condition (Larsen et al., 2007) needs to be satisfied

$$
\operatorname{Var}\left(v_{0}(t)\right)=\sigma_{v_{0}(t)}^{2}=\frac{A_{0}^{2}}{2}
$$

which can be satisfied by defining the filter variable as

$$
\alpha=\Omega_{0}\left(-\zeta_{f}+\sqrt{\zeta_{f}^{2}+\frac{\zeta_{f} \Omega_{0}^{3} A_{0}^{2}}{\pi S_{0}-\zeta_{f} \Omega_{0}^{3} A_{0}^{2}}}\right)
$$

If the standard Wiener process and the vectors of drift and diffusion are denoted by $W(t)$, $\mathbf{c}(\mathbf{Y}(t), t)$ and $\mathbf{d}$, the second-order differential equations of motions can be replaced by the first-order stochastic differential equations in accordance with the formula

$$
d \mathbf{Y}(t)=\mathbf{c}(\mathbf{Y}(t), t) d t+\mathbf{d} d W(t)
$$

where the augmented state vector is given by the following form

$$
\mathbf{Y}(t)=\left[q_{r}(t), \dot{q}_{r}(t), u_{M}(t), \dot{u}_{M}(t), v_{0}(t), \dot{v}_{0}(t), X(t)\right]^{\mathrm{T}}
$$

and the diffusion vector is adopted as

$$
\mathbf{d}=\left[0,0,0,0,0,0, \alpha \sqrt{2 \pi S_{0}}\right]
$$

Using Eqs.(2.19) and (3.1), the components of the drift vector are obtained in the form as

$$
\begin{aligned}
& c_{1}(\mathbf{Y}(t))=\dot{q}_{r}(t) \\
& c_{2}(\mathbf{Y}(t))=-C_{r}(\tau) \dot{q}_{r}(t)-\lambda_{r}^{2}(\tau)\left[\widehat{c}_{r}^{2}(\tau)+\bar{c}^{2}(\tau)-V^{2}(\tau)+\frac{L(\tau)}{2}(a(\tau)-g)\right. \\
& \left.+c^{2}(\tau)\left(\frac{u_{M}(t)}{L(\tau)}+\frac{1}{2}\left(\gamma^{(1)}(\tau)\right)^{2} v_{0}^{2}(t)\right)\right] q_{r}(t)-\frac{\lambda_{r}^{4}(\tau)}{4} c^{2}(\tau) q_{r}^{3}(t) \\
& -\frac{2}{L(\tau)}\left[g \gamma^{(1)}(\tau) \chi_{r}(\tau)-\gamma^{(2)}(\tau) \Omega_{0}^{2}\right] v_{0}(t) \\
& -\frac{4}{L(\tau)}\left[V(\tau) \gamma^{(1)}(\tau) \chi_{r}(\tau)-\gamma^{(2)}(\tau) \zeta_{f} \Omega_{0}\right] \dot{v}_{0}(t)-\frac{2}{L(\tau)} \gamma^{(2)}(\tau) X(t) \\
& c_{3}(\mathbf{Y}(t))=\dot{u}_{M}(t) \\
& c_{4}(\mathbf{Y}(t))=-2 \zeta_{M} \omega_{M}(\tau) \dot{u}_{M}(t)-\omega_{M}^{2}(\tau) u_{M}(t)-\frac{E A}{M} \frac{\lambda_{r}^{2}(\tau)}{4} q_{r}^{2}(t)-\frac{E A}{2 M}\left(\gamma^{(1)}(\tau)\right)^{2} v_{0}^{2}(t) \\
& c_{5}(\mathbf{Y}(t))=\dot{v}_{0}(t) \\
& c_{6}(\mathbf{Y}(t))=X(t)-2 \zeta_{f} \Omega_{0} \dot{v}_{0}(t)-\Omega_{0}^{2} v_{0}(t) \\
& c_{7}(\mathbf{Y}(t))=-\alpha X(t)
\end{aligned}
$$

where the significant change in comparison to the string model presented by Weber et al. (2019) can be seen in the element $c_{2}(\mathbf{Y}(t))$, where the bending stiffness is included.

\section{Solution via equivalent linearization technique}

The application of the equivalent linearization technique into the analysis requires application of the centralized state vector assumed as

$$
\mathbf{Y}^{0}(t)=\left[Y_{1}^{0}(t), Y_{2}^{0}(t), Y_{3}^{0}(t), Y_{4}^{0}(t), Y_{5}^{0}(t), Y_{6}^{0}(t), Y_{7}^{0}(t)\right]
$$


whose particular components are obtained in accordance to the following equations

$$
\begin{aligned}
& Y_{1}^{0}(t)=q_{r}(t)-\mu_{q_{r}}(t) \\
& Y_{2}^{0}(t)=\dot{q}_{r}(t)-\mu_{\dot{q}_{r}}(t) \\
& Y_{3}^{0}(t)=u_{M}(t)-\mu_{u_{M}}(t) \\
& Y_{4}^{0}(t)=\dot{u}_{M}(t)-\mu_{\dot{u}_{M}}(t) \\
& Y_{5}^{0}(t)=v_{0}(t)-\mu_{v_{0}}(t) \\
& Y_{6}^{0}(t)=\dot{v}_{0}(t)-\mu_{\dot{v}_{0}}(t) \\
& Y_{7}^{0}(t)=X(t)-\mu_{X}(t)
\end{aligned}
$$

Equation (3.4) expressed in terms of the centralized state vector is given by the formula

$$
d \mathbf{Y}^{0}(t)=\mathbf{c}^{0}\left(\mathbf{Y}^{0}(t), t\right) d t+\mathbf{d}(t) d W(t)
$$

where the centralized drift vector $\mathbf{c}^{0}\left(\mathbf{Y}^{0}(t), t\right)$ is defined by

$$
\mathbf{c}^{0}\left(\mathbf{Y}^{0}(t), t\right)=\mathbf{c}\left(\mathbf{Y}^{0}(t), t\right)-\mathrm{E}\left[\mathbf{c}\left(\mathbf{Y}^{0}(t), t\right)\right]
$$

and the expressions for the expected values are assumed in the form of differential equations

$$
\frac{d}{d t} \boldsymbol{\mu}(t)=\mathrm{E}\left[\mathbf{c}\left(\mathbf{Y}^{0}(t)\right)\right] \quad \text { with } \quad \boldsymbol{\mu}(t)=\mathrm{E}[\mathbf{Y}(t)]
$$

The elements of the centralized drift vector are described by the formulae

$$
\begin{aligned}
& c_{1}^{0}\left(\mathbf{Y}^{0}(t)\right)=Y_{2}^{0}(t) \\
& c_{2}^{0}\left(\mathbf{Y}^{0}(t)\right)=-C_{r}(\tau) Y_{2}^{0}(t)-\frac{\lambda_{r}^{4}(\tau)}{4} c^{2}(\tau)\left[\left(Y_{1}^{0}(t)\right)^{3}+3\left(Y_{1}^{0}(t)\right)^{2} \mu_{q_{r}}(t)\right. \\
& \left.\quad-3 \operatorname{Var}\left(Y_{1}^{0}(t), Y_{1}^{0}(t)\right) \mu_{r}(t)+3 Y_{1}^{0}(t) \mu_{r}(t)^{2}\right]-\lambda_{r}^{2}(\tau)\left[\widehat{c}_{r}^{2}(\tau)+\bar{c}^{2}(\tau)-V^{2}(\tau)\right. \\
& \left.\quad+\frac{L(\tau)}{2}(a(\tau)-g)\right]\left(Y_{1}^{0}(t)\right)-\frac{2}{L(\tau)}\left[g \gamma^{(1)}(\tau) \chi_{r}(\tau)-\gamma^{(2)}(\tau) \Omega_{0}^{2}\right] Y_{5}^{0}(t) \\
& \quad-\frac{\lambda_{r}^{2}(\tau) c^{2}(\tau)}{L(\tau)}\left[Y_{3}^{0}(t) Y_{1}^{0}(t)-\operatorname{Cov}\left(Y_{1}^{0}(t), Y_{3}^{0}(t)\right)+Y_{1}^{0}(t) \mu_{u_{M}}(t)+Y_{3}^{0}(t) \mu_{q_{r}}(t)\right] \\
& \quad-\frac{\lambda_{r}^{2}(\tau) c^{2}(\tau)}{2}\left(\gamma^{(1)}(\tau)\right)^{2}\left[\left(Y_{5}^{0}(t)\right)^{2} Y_{1}^{0}(t)+\left(Y_{5}^{0}(t)\right)^{2} \mu_{q_{r}}(t)\right. \\
& \left.\quad-\operatorname{Var}\left(Y_{5}^{0}(t), Y_{5}^{0}(t)\right) \mu_{q_{r}}(t)+2 Y_{5}^{0}(t) Y_{1}^{0}(t) \mu_{v_{0}}(t)\right]-\frac{\lambda_{r}^{2}(\tau) c^{2}(\tau)}{2}\left(\gamma^{(1)}(\tau)\right)^{2} \\
& \left.\quad-\quad-2 \operatorname{Cov}\left(Y_{1}^{0}(t), Y_{5}^{0}(t)\right) \mu_{v_{0}}(t)+2 Y_{5}^{0}(t) \mu_{v_{0}}(t) \mu_{q_{r}}(t)+Y_{1}^{0}(t) \mu_{v_{0}}^{2}(t)\right] \\
& \quad-\frac{4}{L(\tau)}\left[V(\tau) \gamma^{(1)}(\tau) \chi_{r}(\tau)-\gamma^{(2)}(\tau) \zeta_{f} \Omega_{0}\right] Y_{6}^{0}(t)-\frac{2}{L(\tau)} \gamma^{(2)}(\tau) Y_{7}^{0}(t) \\
& c_{3}^{0}\left(\mathbf{Y}^{0}(t)\right)=Y_{4}^{0}(t) \\
& c_{4}^{0}\left(\mathbf{Y}^{0}(t)\right)=-\frac{E A}{M} \frac{\lambda_{r}^{2}(\tau)}{4}\left[\left(Y_{1}^{0}(t)\right)^{2}-\operatorname{Var}_{1}\left(Y_{1}^{0}, Y_{1}^{0}\right)+2 Y_{1}^{0}(t) \mu_{q_{r}}(t)\right]-\omega_{M}^{2}(\tau) Y_{3}^{0}(t) \\
& \quad-2 \zeta_{M} \omega_{M}(\tau) Y_{4}^{0}(t)-\frac{E A}{2 M}\left(\gamma^{(1)}(\tau)\right)^{2}\left[\left(Y_{5}^{0}(t)\right)^{2}-\operatorname{Var}_{5}\left(Y_{5}^{0}(t), Y_{5}^{0}(t)\right)+2 Y_{5}^{0}(t) \mu_{v_{0}}(t)\right] \\
& c_{5}^{0}\left(\mathbf{Y}^{0}(t)\right)=Y_{6}^{0}(t) \\
& c_{6}^{0}\left(\mathbf{Y}^{0}(t)\right)=-\Omega_{0}^{2} Y_{5}^{0}(t)-2 \zeta_{f} \Omega_{0} Y_{6}^{0}(t)+Y_{7}^{0}(t) \\
& c_{7}^{0}\left(\mathbf{Y}^{0}(t)\right)=-\alpha Y_{7}^{0}(t)
\end{aligned}
$$

where, like in Eqs. (3.7), the significant difference in comparison to the string model can be seen in the second term. The original non-linear system governed by Eq. (4.2) is replaced with a linear one governed by the equations

$$
d \mathbf{Y}^{0}(t)=\mathbf{B} \mathbf{Y}^{0}(t) d t+\mathbf{d} d W(t)
$$


where the centralized drift terms are assumed as linear functions of the centralized state variables

$$
c_{i, e q}^{0}\left(\mathbf{Y}^{\mathbf{0}}(t)\right)=B_{i m} Y_{m}^{0}
$$

and $B_{i m}$ are the equivalent coefficients determined from the condition that the error (the equation difference) $\varepsilon\left(\mathbf{Y}^{0}\right)$, i.e. the difference between Eqs. (4.2) and (4.6)

$$
\varepsilon\left(\mathbf{Y}^{0}\right)=\mathbf{c}^{0}\left(\mathbf{Y}^{0}\right)-\mathbf{B} \mathbf{Y}^{0}
$$

be minimized in the mean-square sense. Hence, the necessary conditions are

$$
\frac{\partial}{\partial B_{i m}} E\left[\varepsilon^{\mathrm{T}}\left(\mathbf{Y}^{0}\right) \varepsilon\left(\mathbf{Y}^{0}\right)\right]=0 \quad \text { or } \quad \frac{\partial}{\partial \mathbf{B}} E\left[\varepsilon^{\mathrm{T}}\left(\mathbf{Y}^{0}\right) \varepsilon\left(\mathbf{Y}^{0}\right)\right]=0
$$

Thus

$$
B_{i m} E\left[Y_{m}^{0} Y_{j}^{0}\right]=E\left[Y_{j}^{0} c_{i}^{0}\left(\mathbf{Y}^{0}\right)\right] \quad \text { or } \quad \mathbf{B R}_{\mathbf{Y}^{0} \mathbf{Y}^{0}}=E\left[\mathbf{c}^{0}\left(\mathbf{Y}^{0}\right) \mathbf{Y}^{0^{\mathrm{T}}}\right]
$$

where $\mathbf{R}_{\mathbf{Y}^{0} \mathbf{Y}^{0}}$ is the covariance matrix of the state variables. As the state vector $\mathbf{Y}^{0}$ of the equivalent linear system is a zero-mean, the jointly distributed Gaussian random vector process, the following identity is used (Atalik and Utku, 1976)

$$
E\left[\mathbf{Y}^{0} \mathbf{c}^{0^{\mathrm{T}}}\left(\mathbf{Y}^{0}\right)\right]=\mathbf{R}_{\mathbf{Y}^{0} \mathbf{Y}^{0}} E\left[\nabla \mathbf{c}^{0^{\mathrm{T}}}\left(\mathbf{Y}^{0}\right)\right]
$$

where

$$
\nabla=\left[\frac{\partial}{\partial Y_{1}^{0}}, \frac{\partial}{\partial Y_{2}^{0}}, \ldots, \frac{\partial}{\partial Y_{n}^{0}}\right]^{\mathrm{T}}
$$

which yields

$$
\mathbf{B}^{\mathrm{T}}=E\left[\nabla \mathbf{c}^{0^{\mathrm{T}}}\left(\mathbf{Y}^{\mathbf{0}}(t)\right)\right] \quad \text { or } \quad B_{i j}=E\left[\frac{\left.\partial c_{i}^{0} \mathbf{Y}^{0}(t)\right)}{\partial Y_{j}^{0}}\right]
$$

The resulting matrix $\mathbf{B}=\left[b_{i j}\right]$ is

$$
\mathbf{B}=\left[\begin{array}{ccccccc}
0 & 1 & 0 & 0 & 0 & 0 & 0 \\
b_{21} & -C_{r}(\tau) & b_{23} & 0 & F_{r}(\tau) & G_{r}(\tau) & b_{27} \\
0 & 0 & 0 & 1 & 0 & 0 & 0 \\
b_{41} & 0 & -\omega_{M}^{2}(\tau) & -2 \zeta_{M} \omega_{M}(\tau) & b_{45} & 0 & 0 \\
0 & 0 & 0 & 0 & 0 & 1 & 0 \\
0 & 0 & 0 & 0 & -\Omega_{0}^{2} & -2 \zeta_{f} \Omega_{0} & 1 \\
0 & 0 & 0 & 0 & 0 & 0 & -\alpha
\end{array}\right]
$$

where

$$
\begin{array}{ll}
b_{21}=-K_{r}(\tau)+D_{r}(\tau) & b_{23}=-\frac{c^{2} \lambda_{r}^{2}(\tau)}{L(\tau)} \mu_{q_{r}}(t) \quad b_{27}=-\frac{2}{L(\tau)} \gamma^{(2)}(\tau) \\
b_{41}=-\frac{E A \lambda_{r}^{2}(\tau)}{2 M} \mu_{q_{r}(t)} & b_{45}=-\frac{E A}{M}\left(\gamma^{(1)}(\tau)\right)^{2} \mu_{v_{0}}(t)
\end{array}
$$


and the following notations are used

$$
\begin{aligned}
& K_{r}(\tau)=-\lambda_{r}^{2}(\tau)\left[\widehat{c}_{r}^{2}(\tau)+\bar{c}^{2}(\tau)-V^{2}(\tau)+\frac{L(\tau)}{2}(a(\tau)-g)\right] \\
& D_{r}(\tau)=-\frac{3 \lambda_{r}^{4}(\tau)}{4} c^{2}(\tau)\left[\operatorname{Var}\left(Y_{1}^{0}(t), Y_{1}^{0}(t)\right)+\mu_{q_{r}}^{2}(t)\right] \\
& -\lambda_{r}^{2}(\tau) c^{2}(\tau)\left[\frac{\mu_{u_{M}}(t)}{L(\tau)}+\frac{\left(\gamma^{(1)}(t)\right)^{2}}{2}\left(\operatorname{Var}\left(Y_{5}^{0}(t), Y_{5}^{0}(t)\right)+\mu_{v_{0}}^{2}(t)\right)\right] \\
& F_{r}(\tau)=-\lambda_{r}^{2}(\tau) c^{2}(\tau)\left(\gamma^{(1)}(\tau)\right)^{2}\left[\operatorname{Cov}\left(Y_{1}^{0}(t), Y_{5}^{0}(t)\right)+\mu_{v_{0}}(t) \mu_{q_{r}}(t)\right] \\
& -\frac{2}{L(\tau)}\left[g \gamma^{(1)}(\tau) \chi_{r}(\tau)-\gamma^{(2)}(\tau) \Omega_{0}^{2}\right] \\
& G_{r}(\tau)=-\frac{4}{L}\left[V(\tau) \gamma^{(1)}(\tau) \chi_{r}(\tau)-\gamma^{(2)}(\tau) \zeta_{f} \Omega_{0}\right]
\end{aligned}
$$

Solving the differential set of equations for the covariance matrix $\mathbf{R}_{\mathbf{Y}^{0} \mathbf{Y}^{0}}=\mathrm{E}\left[\mathbf{Y}^{0} \mathbf{Y}^{0^{\mathrm{T}}}\right]$ defined by

$$
\frac{d}{d t} \mathbf{R}_{\mathbf{Y}^{0} \mathbf{Y}^{0}}=\mathbf{B R}_{\mathbf{Y}^{0} \mathbf{Y}^{0}}+\mathbf{R}_{\mathbf{Y}^{0} \mathbf{Y}^{0}} \mathbf{B}^{\mathrm{T}}+\mathbf{d d}^{\mathrm{T}}
$$

together with the differential equations for the mean values given by Eq. (4.4) leads to variances and covariances of particular random state variables.

\section{Numerical examples - results and discussion}

To examine the influence of cable bending stiffness on the final results of a particular random state variable in comparison to the string model, the numerical analysis was carried out for the same data as used by Weber et al. (2019): the total height of the system $Z_{0}=243 \mathrm{~m}$, the travel height $H=200 \mathrm{~m}$ and the initial length of the cable $L(0)=7.5 \mathrm{~m}$. The main mass $M=500 \mathrm{~kg}$ is moving downwards with the transport speed $V=3.5 \mathrm{~m} / \mathrm{s}$. The external excitation of the host structure is assumed to have frequency $\Omega_{0}=0.25 \mathrm{~Hz}$ and amplitude at the top level $A_{0}=0.3 \mathrm{~m}$. The damping ratios used in the analysis are $\zeta_{M}=0.03$ and $\zeta_{r}=0.03$. The cable mass per unit length is $m=1.8 \mathrm{~kg} / \mathrm{m}$, and the longitudinal stiffness is $E A=15.1 \mathrm{MN}$.

The value of the bending stiffness of the cable to be taken into account during the numerical computation needs careful consideration. The bending stiffness depends on the modulus of elasticity of SWRs and tension of the cable. Therefore, it can not be directly calculated by using the cross-section moment of inertia. In most cases, the value of bending stiffness $E I$ is determined experimentally. The numerical analysis carried out here is for two assumed values, namely for $15 \mathrm{Nm}^{2}$ and $80 \mathrm{Nm}^{2}$, respectively (Kaczmarczyk and Mirhadizadeh, 2016). The simulations carried out yield similar results for these values of bending stiffness. The results for $E I=80 \mathrm{Nm}^{2}$ are compared with the string model (Weber et al., 2019), and the corresponding results obtained from the MC simulation are presented. Figure 2a shows that differences between the mean (expected) values of the generalized coordinates $q_{r}$ obtained from the two models are insignificant. On the other hand, some differences in the expectations of vertical displacements $u_{M}$ of the main mass (Fig. 2b) as well as in the variances of $q_{r}$ and $u_{M}$ (Figs. 2c and 2d) obtained from the two models can be observed.

The results for the model with bending stiffness obtained by the application of the equivalent linearization technique depend on the damping ratio of the auxiliary damping filter $\zeta_{f}$ applied in the analysis. The plots shown in Fig. 3 demonstrate that the lower the value of $\zeta_{f}$, the smaller 
(a)

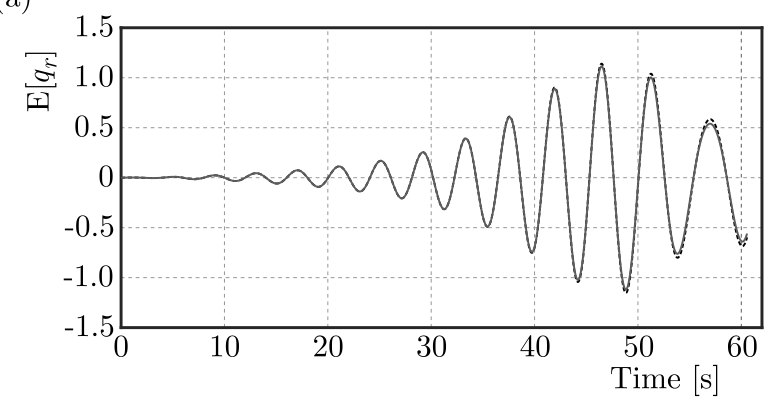

(c)

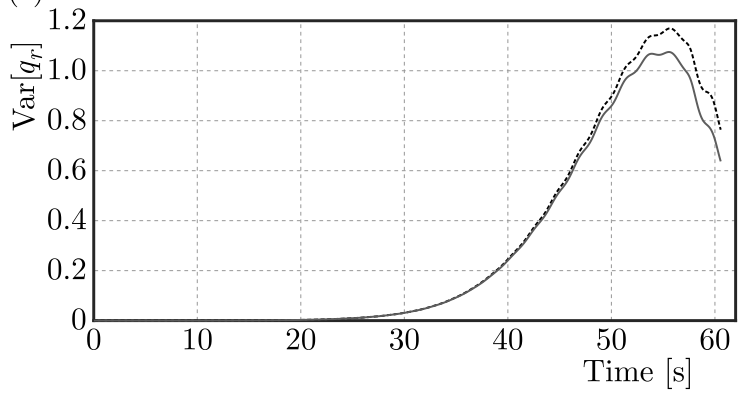

(b)

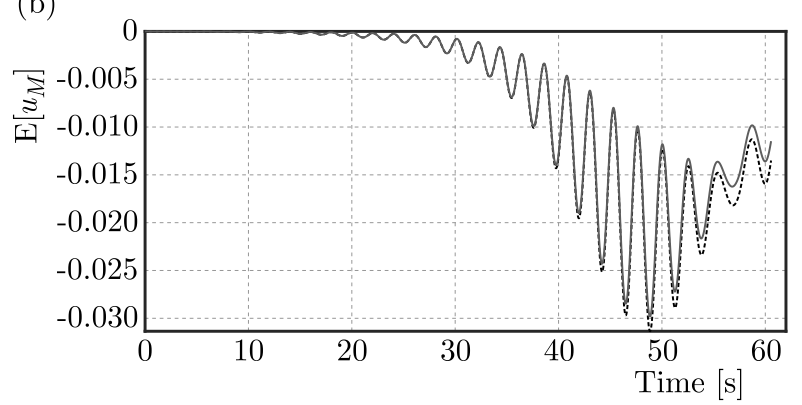

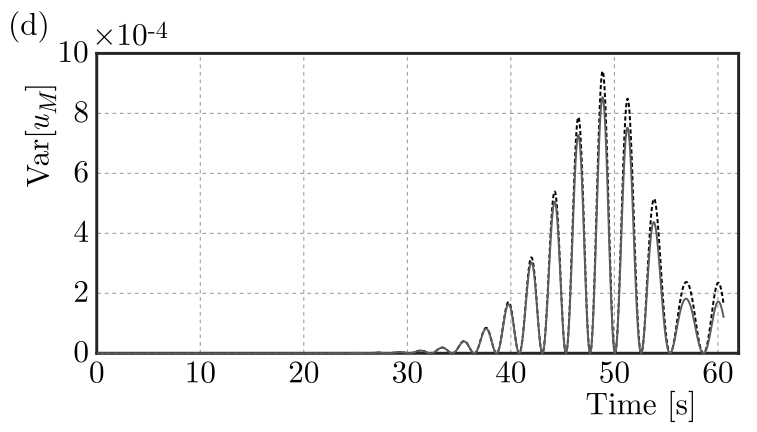

Fig. 2. Expected values and variances of a particular random state variable

(a)

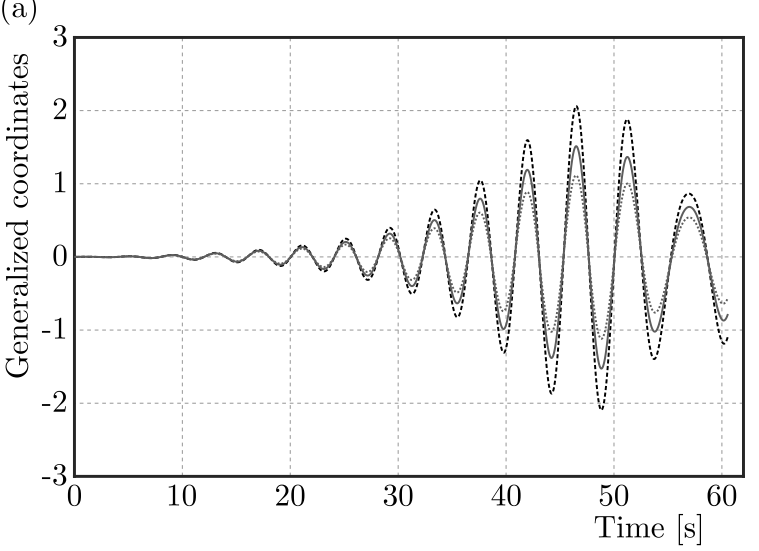

(b)

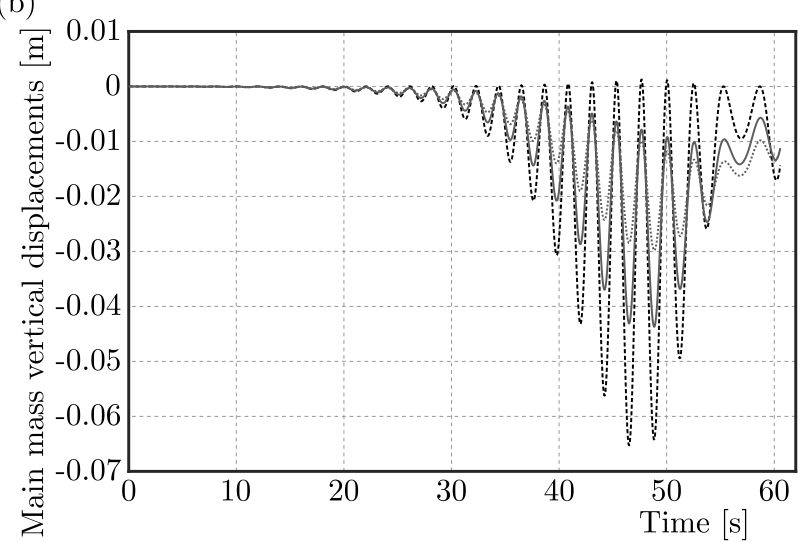

…-... Nonlinear deterministic results

- Equivalent linearization technique $\left(\zeta_{f}=0.005\right)$

.......... Equivalent linearization technique $\left(\zeta_{f}=0.010\right)$

Fig. 3. Comparison of nonlinear deterministic results and expected values

the differences between the deterministic and stochastic solution. The same regularity was also observed for the string model (see Weber et al., 2019).

To verify the results obtained by the equivalent linearization technique, Monte Carlo simulation was used and conducted by using 2000 simulations for the time step $\Delta t=0.025 \mathrm{~s}$. The calculation was made only for one value of the coefficient $\zeta_{f}=0.01$, for which the condition given by Eq. (3.2) was satisfied. The same as in the string model, the expected values of generalized coordinates and variances of $v_{0}$ and $X(t)$ showed good matches between the curves (see Weber et al., 2019). The expected values of vertical displacements of the main mass and variances of $u_{M}$ and $q_{r}$ showed greater differences between both methods but the order of magnitudes presented on the diagrams were comparable. It brings a conclusion that to obtain satisfactory convergence 
of the results from both methods, the number of simulations in $\mathrm{MC}$ computations should be near 10000 , but then the cost of the calculation is very high.

(a)

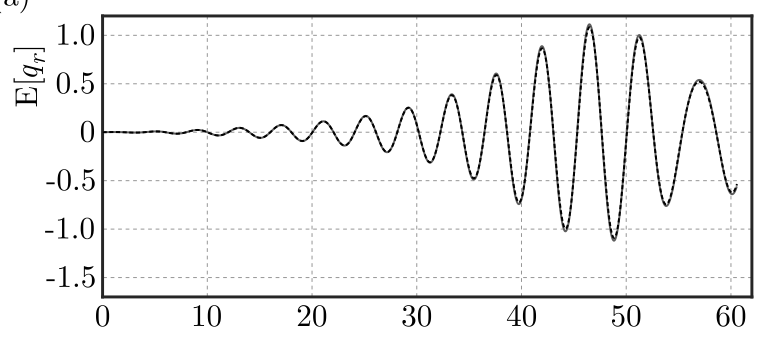

(b)

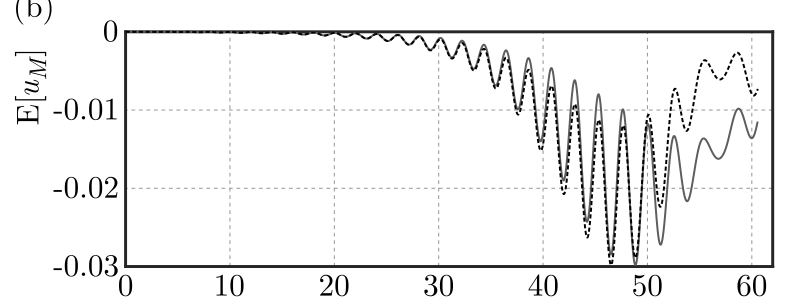

(c)

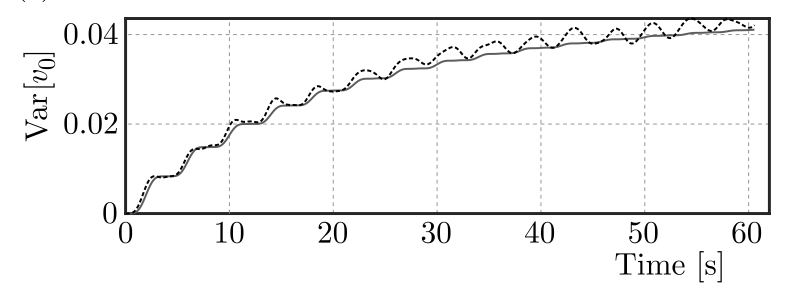

(d)

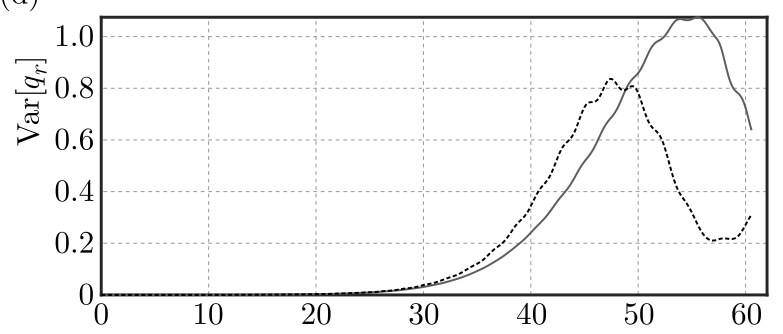

(e)

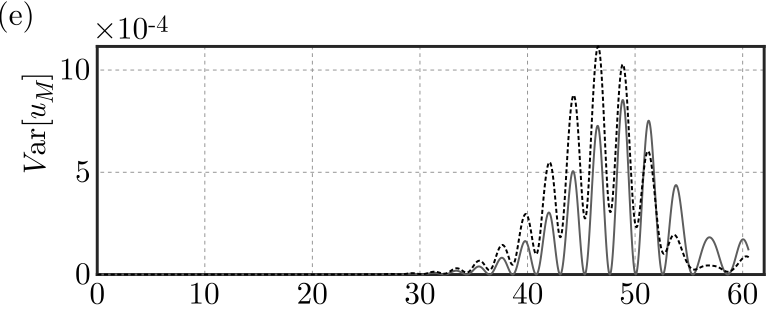

(f)

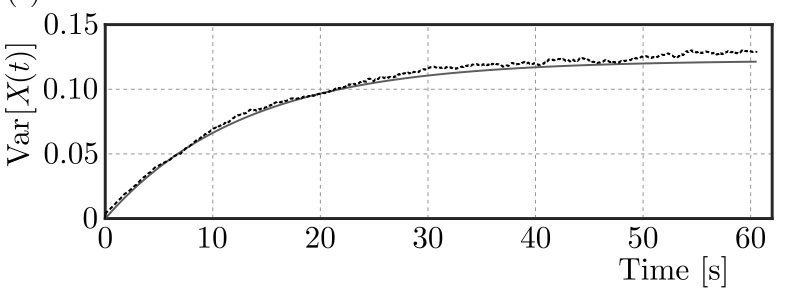

- Equivalent linearization technique $\left(\zeta_{f}=0.01\right) \quad \cdots$... Monte Carlo simulation

Fig. 4. Results of the equivalent linearization technique and Monte Carlo simulation

The comparison of the system fundamental frequency shows that for the string model, the $\omega_{r}$ reaches the value $0.25 \mathrm{~Hz}$ for length $L=132.9$, whereas for the model including cable bending stiffness, the resonance effect can be observed for the length equal to $144 \mathrm{~m}$. Precise determination of the resonance point is very important in the analysis of dynamic behaviour of the system and when suitable resonance mitigating measures, such as application of tuned mass damper systems, need to be considered.

\section{Concluding remarks}

The results presented in this paper demonstrate that the behaviour of the system represented by the taut string model of the cable and by the model with cable bending stiffness is similar. This is due to a relatively small bending stiffness and high axial tensions of SWRs that are applied in the calculations. However, some differences are noticeable in the expected values of vertical displacements of the main mass and its variances.

Additionally, it should be noted that a significant shift of the resonance point can be observed after considering the bending stiffness during the analysis, which is very important for safe design and evaluation of structural integrity of traction driven transportation systems deployed in high-rise buildings.

In conclusion, the taut string model can be used in the analysis of the cable-mass system moving in a cantilever host structure under the operating conditions considered, when the axial tension of the cable is large. For lower axial tension values and slack cable conditions, the tensioned beam model should be considered. In such cases, further analysis would be required 
to accommodate more involved boundary conditions applied at the lower and upper ends of the cable, respectively.

\section{References}

1. Atalik T.S., UtKu S., 1976, Stochastic linearization of multi-degree-of-freedom nonlinear systems, Earthquake Enginering Structures Dynamics, 4, 411-420

2. Caughey T.H., 1963, Equivalent linearization techniques, Journal of the Acoustical Society of America, 35, 1706-1711

3. Evan-Iwanowski R.M., 1976, Resonance Oscillations in Mechanical Systems, Elsevier Scientific Publishing Company

4. Giaccu G.F., Barbiellini B., Caracoglia L., 2015, Stochastic unilateral free vibration of an in-plane cable network, Journal of Sound and Vibration, 340, 95-111

5. Kaczmarczyk S., Iwankiewicz R., 2017a, Gaussian and non-Gaussian stochastic response of slender continua with time-varying length deployed in tall structures, International Journal of Mechanical Sciences, 134, 500-510

6. Kaczmarczyk S., Iwankiewicz R., 2017b, On the nonlinear deterministic and stochastic dynamics of a cable - mass system with time-varying length, 12th International Conference on Structural Safety and Reliability, Austria, 1205-1213

7. Kaczmarczyk S., Iwankiewicz R., Terumichi Y., 2009, The dynamic behaviour of a nonstationary elevator compensating rope system under harmonic and stochastic excitations, Journal of Physics: Conference Series, 181, 12-47

8. KaczmarczyK S., Mirhadizadeh S., 2016, Quasi-stationary mechanics of elastic continua with bending stiffness wrapping on a pulley system, Journal of Physics: Conference Series, 721, 012011, $1-7,1742-6588$

9. Kijewski-Correa T., Pirnia D., 2007, Dynamic behavior of tall buildings under wind: in-sights from full-scale monitoring, The Structural Design of Tall Special Buildings, 16, 471-486

10. Kougioumtzoglou I.A., Fragkoulis V.C., Pantelous A.A., Pirotta A., 2017, Random vibration of linear and nonlinear structural systems with singular matrices: A frequency domain approach, Journal of Sound and Vibration, 404, 84-101

11. Larsen J.W., Iwankiewicz R., Nielsen S.R.K., 2007, Nonlinear stochastic stability analysis of wind turbine wings by Monte Carlo simulations, Probabilistic Engineering Mechanics, 22, 181-193

12. MitropolskiI Y.A., 1965, Problems of the Asymptotic Theory of Nonstationary Vibrations, Israel Program for Scientific Translations Ltd, Jerusalem

13. Proppe C., Pradlwarter H.J., Schü̈ller G.I., 2003, Equivalent linearization and Monte Carlo simulation in stochastic dynamics, Probabilistic Engineering Mechanics, 18, 1-15

14. Roberts J.B., 1981, Response of non-linear mechanical systems to random excitations. Part II: Equivalent linearization and other methods, Shock and Vibration Digest, 13, 15-29

15. Roberts J.B., Spanos P.D., 1990, Random vibration and statistical linearization, John Wiley \& Sons

16. Socha L., 2008, Linearization Methods for Stochastic Dynamic systems, Lecture Notes in Physics, 730, Springer

17. Spanos P.D., 1981, Stochastic linearization in structural dynamics, Applied Mechanics Reviews, ASME, 34, 1-8

18. Spanos P.D., Evangelatos G.I., 2010, Response of nonlinear system with restoring forces governed by fractional derivatives - Time domain simulation and statistical linearization solution, Soil Dynamics and Earthquake Engineering, 30, 811-821 
19. Terumichi Y., Ohtsuka M.,Yoshizawa M., Fukawa Y., Tsujioka Y., 1995, Nonstationary vibrations of a string with time-varying length and a mass-spring system attached at the lower end, Nonlinear Dynamics, 12, 39-55

20. Thompson W.T., 1993, Theory of vibration with Applications Fourth Edition, Chapman and Hall, University and Professional Division, London

21. Weber H., Iwankiewicz R., Kaczmarczyk R., 2019, Equivalent linearization technique in nonlinear stochastic dynamics of a cable-mass system with time-varying length, Archives of Mechanics, 71, 393-416

Manuscript received November 30, 2019; accepted for print February 24, 2020 